\title{
Mechanically Robust Hydrophobized Double Network Hydrogels for Water Purification
}

\author{
Marshall J. Allen ${ }^{1,2 \S}$, Rahul Sujanani ${ }^{2 \S}$, Alyssa Chamseddine ${ }^{2}$, Benny D. Freeman ${ }^{2}$, and \\ Zachariah A. Page ${ }^{1 *}$ \\ $\S$ These authors contributed equally to this work
}

*To whom correspondence should be addressed: zpage@utexas.edu

1. Department of Chemistry, The University of Texas at Austin, Austin, TX 78712, USA

E-mail: zpage@utexas.edu

2. McKetta Department of Chemical Engineering, The University of Texas at Austin, Austin, TX, 78712, USA

Keywords: double network hydrogel, interpenetrating polymer network, ion exchange membrane, water purification

Abstract: Water swollen polymer networks are attractive for applications ranging from tissue regeneration to water purification. For water purification, charged polymers provide excellent ion separation properties. However, many ion exchange membranes (IEMs) are brittle, necessitating the use of thick support materials that ultimately decrease throughput. To this end, a series of double network hydrogels (DNHs), synthesized with varied composition to decrease water content, are examined as robust membrane materials for water purification. One network contains fixed anionic charges, while the other comprises a copolymer with different ratios of hydrophobic ethyl acrylate (EA) and hydrophilic dimethyl acrylamide (DMA) repeat units. Characterizing water content and mechanical performance in free standing DNH films reveals a $\sim 5 \times$ decrease in water content, while increasing ultimate stress and strain by $\sim 3.5 \times$ and $\sim 4.5 \times$ for 90:5 EA:DMA relative to pure DMA. Salt transport properties relevant to water purification, including permeability, solubility, and diffusivity, are measured and show improved performance upon reducing water content. Overall, the ability to simultaneously reduce water content, increase mechanical integrity, and decrease salt transport rates highlights the potential of DNHs for membrane applications. 


\section{Introduction}

Interpenetrating polymer networks (IPNs) are a versatile class of soft materials that merge attractive properties (e.g., mechanical, optical, and electrical) to enable applications in tissue engineering, structural automotive and aerospace components, and separation systems ${ }^{1,2,3,4}$. IPNs are defined as polymer blends in which at least one network is crosslinked or polymerized within the other, such that separating the networks cannot occur without breaking covalent chemical bonds. In 2003, Gong and coworkers ${ }^{5}$ introduced a special class of IPNs, termed double networks, which synergistically combine rigid and soft networks to provide mechanical properties superior in performance relative to the sum of each individual network. While the mechanical properties of double network IPNs are exceptional, their composition and associated functionality remain narrow in scope, primarily localized to the biomedical field ${ }^{6,7,8}$.

To-date, double network architectures have enabled the preparation of tough, ductile polymeric materials with tissue-like softness (Young's modulus $(E) \approx 100-1000 \mathrm{kPa})^{6}$. DNHs exhibit remarkable toughness, $\sim 10^{2}-10^{3} \mathrm{~J} \mathrm{~m}^{-2}$ relative to $\sim 10 \mathrm{~J} \mathrm{~m}^{-2}$ for analogous single network hydrogels ${ }^{9}$. Critical to their mechanical performance is the combination of a minority rigid network, embedded in a soft majority network, typically in ratios of $\sim 1: 20^{6}$. While this architecture has been demonstrated for both hydrogels (wet) and elastomers (dry), double network hydrogels (DNHs) have dominated the literature due to their resemblance to biological tissue and thus relevance to regenerative medicine applications ${ }^{6,7,8,10}$. While the inherently high-water content (>90 wt\%) of DNHs has facilitated their utility in the field of biomaterials, it has limited their applicability in others, including as water purification membranes. Although water purification membranes are also hydrated, water content typically ranges from $\sim 10-60 \mathrm{wt} \%^{11}$ to balance throughput and selectivity for a desired species. For example, desalination membranes with higher degrees of water sorption tend to provide higher water flux or permeability, but less discrimination 
between water and salt ${ }^{12}$. Broadening the application of DNHs to examine their utility as robust water purification membranes would require fundamental understanding of how: 1) water content can be controlled without compromising mechanical performance and 2) ion transport is impacted by network composition.

In traditional DNH syntheses a rigid, highly crosslinked, and charged first network is generated using free radical polymerization and subsequently swollen with an aqueous solution containing the second monomer, which provides a more ductile, lightly crosslinked network ${ }^{9}$. Polymerization of the second monomer then traps the first network in its swollen, or pre-stretched state (Figure 1). Critically, the fixed charge moieties of the first network facilitate swelling by drawing water into the gel osmotically and requiring counter-ions to maintain electroneutrality ${ }^{13}$. In its final form, the pervasive, rigid first network acts like rebar in concrete, reinforcing, or toughening, the DNH by distributing stress throughout the soft matrix and dissipating energy upon breaking under strain ${ }^{6}$. While successful, this method necessitates a pre-swollen/stretched first network, limiting the chemical composition of the second network to one that can effectively sorb into and swell the first network ${ }^{7}$. Commonly, DNHs pair poly(2-acrylamido-2-methyl-1propanesulfonic acid) (PAMPS) or poly(3-sulfopropyl acrylate potassium salt) (PSAPS) as the ionic first network with a similarly hydrophilic poly(acrylamide) or poly(ethylene glycol) second network ${ }^{5}$. The presence of fixed charge moieties in DNHs also makes them potentially attractive candidates as ion exchange membranes (IEMs), which are applied in water purification due to their ability to provide ion selectivity ${ }^{14}$. However, the high-water content of traditional DNHs inherently dilutes charge density and reduces selectivity, precluding their use as membrane materials. 

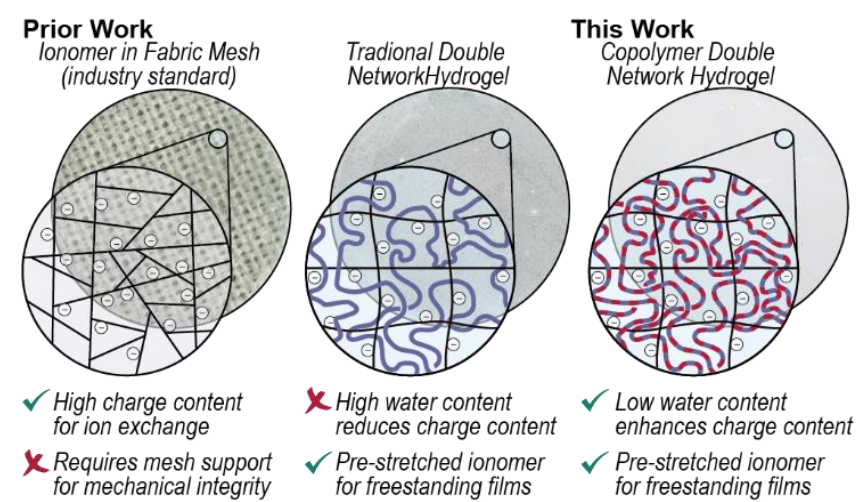

Figure 1. Comparison of conventional ion exchange membranes (IEMs) used for water purification and double network hydrogels (DNHs) used for biomedical applications with the present, hydrophobized DNHs prepared in this study. Current IEMs often utilize high crosslink densities to reduce water content, which results in brittle membranes that require a thick, fabric mesh for mechanical support. In contrast, DNHs are free standing with fixed charges incorporated into the backbone yet have substantially high-water contents (>90 wt\%), precluding their utility as IEMs. This work investigates a new synthetic route to reduce water content in an effort to explore DNHs as potential IEM materials.

The ideal IEM is mechanically robust in operation and contains a high concentration of fixed charge groups, as these groups act to reject salt in osmotic processes (e.g., reverse osmosis and nanofiltration) and provide high counter-ion selectivity in electrically driven processes (e.g., electrodialysis $)^{15}$. These characteristics are often at odds with one another, where membrane brittleness is the inevitable result of using high crosslink densities to suppress swelling caused by high charge content (Figure 1). The contemporary industrial solution has been to fabricate a highly crosslinked and brittle ion exchange resin within the pores of a thick (e.g., $600 \mu \mathrm{m}$ ), fabric mesh for mechanical support. This mechanical support increases the composite membrane thickness, thereby reducing flux and limiting efficiency. This is also a pervasive challenge for many flatsheet membrane modules, which utilize thin active layers for separation that are mechanically supported by a porous structure. Therefore, the ability to generate tough, free-standing, thin films with desired functionality (e.g., high fixed charge density for IEMs) would represent a significant and broad advance in polymer materials for membrane technology.

One method to enable the application of robust DNHs as IEMs is to develop synthetic pathways to reduce water content, which would increase fixed charge concentrations and 
selectivity of DNHs. Hydrophobic elastomers with double network architectures were recently reported by Creton and coworkers ${ }^{16}$, providing precedent for achieving mechanical integrity without the need for a final solvated state. In this report, a hydrophobic poly(ethyl acrylate) first network was swollen with methyl acrylate, which was subsequently polymerized to form the double network structure. However, no fixed charge groups were present in these double network elastomers. Alternatively, Gong and coworkers ${ }^{17}$ recently demonstrated that using solvents with higher dielectric constants than water can enable the introduction of more hydrophobic second networks into charged, hydrophilic first networks to generate double network elastomers. In their work, $N$-methylformamide (NMF, dielectric constant $\approx 170$ vs 80 for water) is used in conjunction with a hydrophobic second monomer (e.g., ethyl, methoxyethyl, and carbitol acrylates) to produce a mixture capable of adequately pre-swelling a charged network of PAMPS, ultimately providing a mechanically tough double network elastomer post-polymerization and removal of solvent. Despite these advances, the extent and impact of water swelling on mechanical and membrane transport properties have yet to be examined. We propose that the use of a hydrophobic second network in a DNH can reduce water content while maintaining mechanical integrity, facilitating potential membrane applications (Figure 1).

In this study, the synthesis of DNHs with reduced water content is described, along with mechanical and transport property characterization to examine, for the first time, their potential utility as IEMs. The first network provides fixed charge groups by using PAMPS, while the second network composition is varied by copolymerizing hydrophilic and hydrophobic monomers to control water content and stabilize this network under the final aqueous conditions for application. The mechanical strength and toughness of the presented DNHs rivals the performance of traditional DNHs and elastomers, while simultaneously reducing salt transport relative to 
conventional DNHs. The results demonstrate the prospect for this synthetic strategy to produce robust, freestanding IEMs.

\section{Results and Discussion}

\subsection{Double Network Hydrogel Preparation}

Double networks with reduced water content were prepared by swelling a charged first network, poly(2-acrylamido-2-methylpropane sulfonic acid) (poly(AMPS)), with $\quad N$ methylformamide (NMF) containing a mixture of a hydrophobic monomer, ethyl acrylate (EA), and a hydrophilic monomer $N, N$-dimethyl acrylamide (DMA). The ratio of EA:DMA was varied between 0 to 1, with samples named based on the percent of EA in the monomer mixture (Table S1). The second network resin was copolymerized in the presence of $N, N^{\prime}-$ methylenebisacrylamide (0.001 equivalents) as a crosslinker, using UV light to activate a radical photoinitiator, $\alpha$-ketoglutaric acid (Figure 2$)$. The high dielectric constant of NMF $(\approx 170$ vs 80 for water) was critical to achieve ample swelling of the first network in the presence of the

hydrophobic monomer, $\mathrm{EA}^{17}$. A more detailed discussion regarding experimental conditions for both networks is presented in the SI (Membrane Synthesis section). 


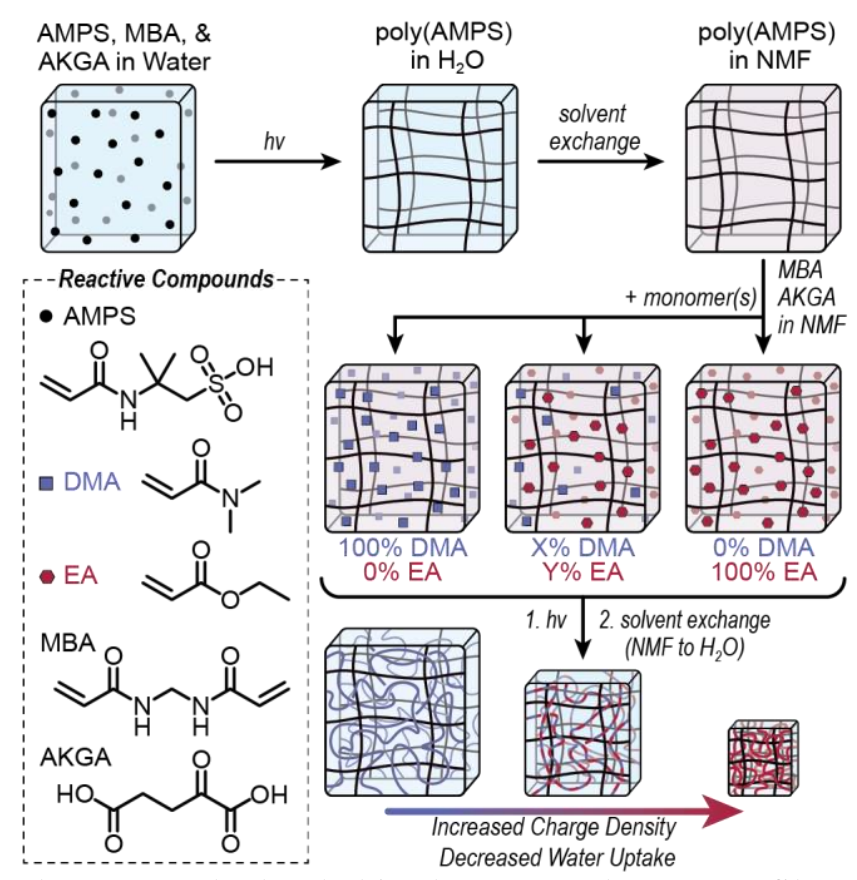

Figure 2. Synthetic method to prepare hydrophobized DNHs. Poly(AMPS) films were cast from an aqueous solution containing initiator $(0.001 \mathrm{eq})$ and crosslinker $(0.04 \mathrm{eq})$. Water in the Poly(AMPS) gels were exchanged to $N$-methylformamide (NMF) before being soaked in a $53 \mathrm{vol} \%$ monomer solution in (NMF). Monomer compositions were varied from $0-100 \%$ ethyl acrylate relative to dimethyl acrylamide. After casting the second network, and transitioning the films back into the aqueous form, increased EA content resulted in reduced water uptake and increased charge density.

\subsection{Water Uptake}

All double network compositions produced qualitatively tough, transparent organogels after irradiation. However, once exchanged and hydrated with DI water, films prepared with greater than 50 vol\% EA displayed translucence, with complete opacity observed for all films prepared with $\geq 90$ vol\% EA (Figure 3a). We hypothesize that opacity, upon hydration, is indicative of macroscopic phase separation (i.e., on an order that scatters visible light), which arises from the collapse of the hydrophobic EA domains outside the poly(AMPS) supported hydrophilic domains. This hypothesis is supported by scanning electron microscopy (SEM) images of freeze-dried DNHs (Figure 3b), where void (i.e., hydrophilic domain) size decreases as EA content is increased. Further details and analysis of void area are found in Figures S7-8 in the SI. 
Equilibrium water uptake of the DNHs (Figure 3c and Table S3) is consistent with increases in second network hydrophobicity, as water content decreased with increasing EA content, plateauing
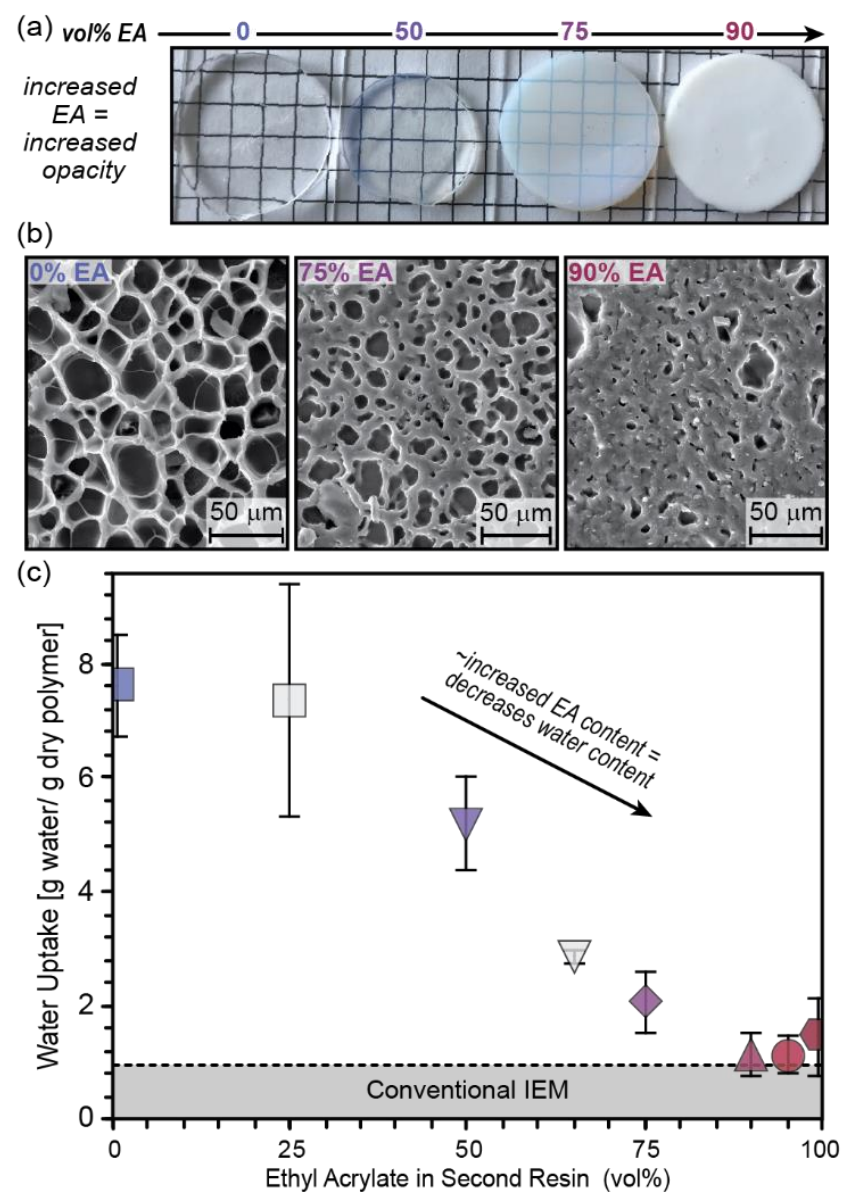

Figure 3. The effect of varying EA:DMA ratio on morphology and water content. (a) Digital images of circular punches from hydrated films, showing an increase in opacity as EA content increases. (b) Representative SEM images showing a decrease in void (i.e., hydrophilic domain) size as EA increases. (c) Mass water uptake as a function of EA content, showing that water content decreases as EA increases and approaches values exhibited by conventional IEMs, as represented by the grey bar $(\leq 1 \mathrm{~g} / \mathrm{g})$. Values and error bars represent the average and standard deviation of one to three different film casts, each with three sample punches per film.

at $\sim 1.1 \mathrm{~g}$ sorbed water/g dry polymer $(\approx 55 \mathrm{wt} \%$ water) for the 90 and $95 \%$ EA samples. Notably, the $100 \%$ EA samples showed a slight increase in water content. From the freeze dried SEM images (Figure S5-6 in the SI), large voids with thick hydrophobic domains suggest partial collapse of the second network in water, which is hypothesized to arise from an incompatibility of poly(EA) with water. Overall, the substantial reductions in water content brought about by the 
presence of a more hydrophobic, yet compatible, second network, provides DNHs with water uptake values significantly closer to the range exhibited by conventional IEMs $(\leq 1 \mathrm{~g} / \mathrm{g}$ or $\leq 50$ wt $\%$ water). Other potential strategies to reduce water content include increasing AMPS concentration during the formation of the first network and increasing crosslinker content in the first network, which were briefly examined in addition to the varied EA:DMA ratio presented here (Figures S12-S13 in the SI).

\subsection{Mechanical Testing}

The influence of network composition on mechanical performance was characterized using tensile testing. Samples were prepared using a standard (ASTM D638) dogbone punch. It was hypothesized that as hydrophobic EA content was increased, mechanical properties would deteriorate. However, this was not observed. Rather, as EA content increased up to $100 \%$, both the stress at fracture $\left(\sigma_{f}\right)$ and strain at fracture $\left(\varepsilon_{f}\right)$ increased, providing higher strain energy density

\section{$\left(U_{0}\right)$ (Figure 4).}

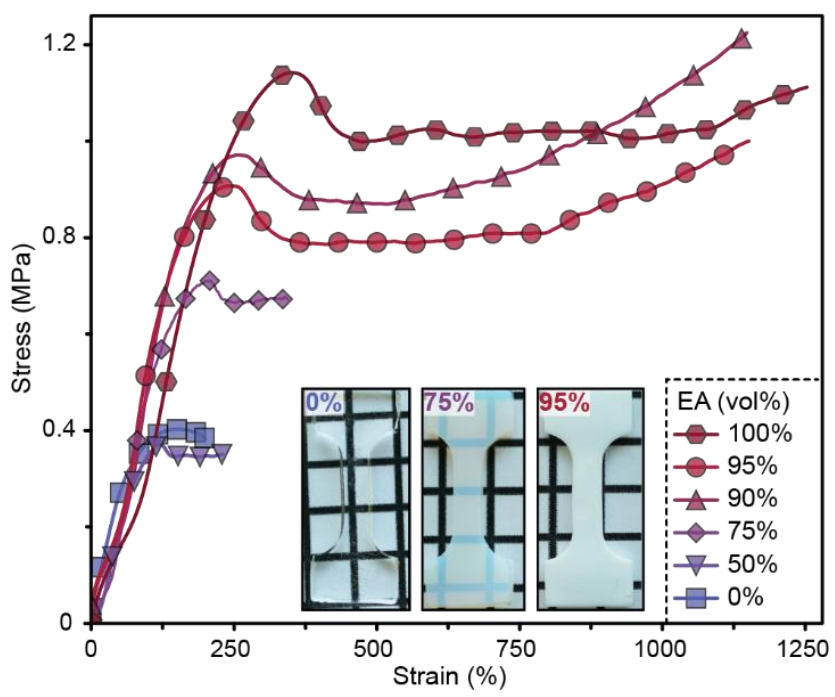

Figure 4. Representative stress-strain curves for hydrated dogbones under uniaxial tension. Both stress and strain at break increased with increasing EA content, with a mechanical response consistent with traditional DNHs. Number next to stress-strain curves are EA contents in vol\%. The inset provides three representative images for 0, 75, and 95 vol\% EA mechanical samples. Traces represent smoothed data to guide the eye. Large "bumps" observed after initial yielding were associated with additional necking events occurring (Figure S4b). 
Similar to the behavior of traditional high-water content DNHs, a plastic-like necking was observed past the yield point for all compositions, along with strain hardening prior to fracture for samples with $\geq 90$ vol\% EA. For samples with $<90$ vol\% EA, strain hardening was not reached, presumably due to the significantly smaller strains at break. The 100 vol\% EA samples had a $U_{0}$ value of $19 \pm 3 \mathrm{MPa}$, surpassing brittle IEM materials such as sulfonated polysulfone ( $U_{0} \sim 2 \mathrm{MPa}$ ) and even relatively strong IEM materials such as Nafion ( $\left.U_{0} \sim 10 \mathrm{MPa}\right)$ (Table 1). Although the DNHs may be considered "tougher" than these traditional IEMs, they are also much more compliant due to their relatively low stiffness, or Young's modulus $(E) ; 0.83 \pm 0.32, \approx 21 \mathrm{MPa}$, and $\approx 200 \mathrm{MPa}$ for DNH (95 vol\% EA), Nafion ${ }^{18}$, and sulfonated polysulfone ${ }^{19,20}$, respectively. As a result of the low stiffness, deformation of the present DNH films may occur under applied pressures, which is an important factor to consider when selecting operational configuration for these materials if employed as water purification membranes. Regardless, the high $U_{0}$ values will decrease the likelihood of fracture and potentially increase the lifetime of these materials as membranes.

Table 1. Representative mechanical properties for present DNH samples contextualized with traditional DNHs (PAMPS/PAAm) and industrially relevant IEM materials.

\begin{tabular}{lcccc}
\hline Membrane Material & $\begin{array}{c}\text { Stress at Break } \\
{[\mathrm{MPa}]}\end{array}$ & $\begin{array}{c}\text { Strain at } \\
\text { Break } \\
{[\%]}\end{array}$ & $\begin{array}{c}\text { Young's } \\
\text { Modulus } \\
{[\mathrm{MPa}]}\end{array}$ & $\begin{array}{c}\text { Strain Energy } \\
\text { Density } \\
{[\mathrm{MPa}]}\end{array}$ \\
\hline 0 mol\% EA DNH & $0.4 \pm 0.1$ & $189 \pm 96$ & $0.6 \pm 0.2$ & $0.5 \pm 0.5$ \\
$95 \mathrm{~mol} \%$ EA DNH & $1.3 \pm 0.3$ & $1350 \pm 330$ & $0.8 \pm 0.3$ & $12 \pm 4$ \\
PAMPS/PAAm DNH & 1 & 1000 & $0.1-1.0$ & 8 \\
Sulfonated Polysulfone $^{19,20}$ & $15-20$ & $5-10 \%$ & $100-300$ & $\sim 2-3$ \\
${\text { Nafion } 115^{18}}^{18}$ & 21 & 115 & 21 & $\sim 10-13$ \\
\hline
\end{tabular}

\subsection{Charge Content}

High performance IEMs derive ion selective properties based on a high density of fixed charge groups (e.g., greater than $1 \mathrm{~mol}$ of fixed charges per liter of sorbed water). As such, charge 
content of the DNHs prepared in this study was estimated using an ion-exchange procedure, with detailed methodology reported in the SI. In short, the concentration of $\mathrm{Na}^{+}$counter-ions in films equilibrated with DI water was determined by using a $\mathrm{CsCl}$ solution to exchange $\mathrm{Na}^{+}$with $\mathrm{Cs}^{+}$, extracting $\mathrm{Na}^{+}$into the solution phase for measurement ${ }^{21}$. As anticipated, increases in membrane fixed charge content correlated well with decreases in water content (i.e., increases in EA content) as shown in Figure 5. Here, fixed charge concentration is reported in units of mol/L sorbed water (i.e., based on the volume of water in the membrane), as this is the pertinent unit for the thermodynamics of ion exclusion ${ }^{21}$. Fixed charge content using other common units including, $\mathrm{mol} / \mathrm{L}$ swollen membrane or meq/g dry polymer, when reported as an ion exchange capacity (IEC), are detailed in the SI (Table S6).

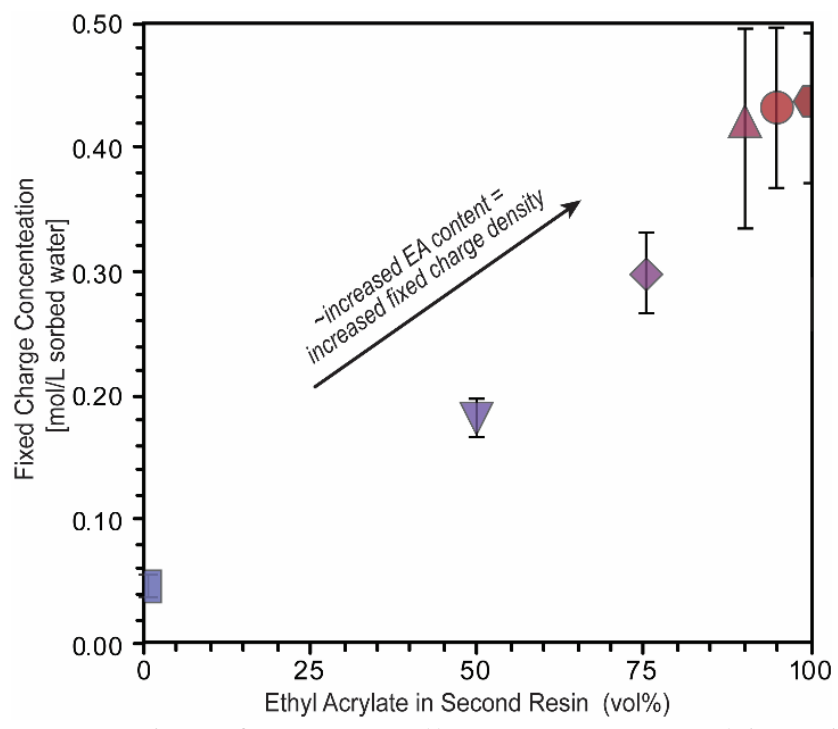

Figure 5. Fixed charge concentration of water swollen DNHs reported in units of mol/L sorbed water plotted as a function of EA content incorporated into the second network precursor solution. As the EA content increases, the charge density of these DNHs increases and is attributed, in part, to a concurrent decrease in water content.

Charge content values for DNHs with 0 and 100 mol\% EA were $0.048 \pm 0.008$ and $0.44 \pm 0.07$ mol/L sorbed water, respectively. To place these values into context, commercial IEMs (e.g., CR61, AR-103, CMX, AMX, etc.) can exhibit fixed charge contents greater than $3 \mathrm{~mol} / \mathrm{L}$ sorbed water $^{22}$, approximately one order of magnitude larger than the values observed for the reduced 
water content DNHs in this study. This represents an opportunity to further advance this new class of membrane materials by either reducing water content further or introducing additional charged groups without compromising mechanical performance.

In addition to water content, the fixed charge content of a DNH is influenced by the relative amounts of charged first network and neutral second network in the final gel, which depends on the ability of the second network to partition into the first, though it is difficult to predict how changes in chemical composition and processing conditions will influence fixed charge density. However, given the synthetic conditions known to yield DNHs with good mechanical properties (i.e., a minority rigid $1^{\text {st }}$ network embedded within a majority soft $2^{\text {nd }}$ network), we hypothesize that the observed low charge content is due to an inherently low fraction of the first anionic network relative to the second neutral network. Gong and coworkers ${ }^{17}$ have shown that the optimal mechanical performance of DN elastomers occurs when the ratio of first to second network is $\sim 2$ to 8 vol\% ${ }^{6,17}$. To support this hypothesis for our DNHs, the mass fraction of the first network was estimated using the measured IEC and was found to range from $\sim 0.08$ to 0.20 for the $0 \%$ EA and $50 \%$ EA, respectively (all other materials fell within this range). These results and calculation details are provided in the SI (Table S6).

\subsection{Salt Transport Properties}

To examine the prospective utility of the DNHs prepared in this study as water purification membranes, salt permeability, salt solubility, and salt diffusivity, which are relevant transport properties for osmotic processes, were measured using a feed solution of $0.1 \mathrm{~mol} / \mathrm{L} \mathrm{NaCl}$ (approximate ionic strength of brackish water ${ }^{23}$. Initially, salt permeability of the DNHs with the lowest water content ( $95 \mathrm{vol} \%$ EA) was found to be $\sim 10^{-9} \mathrm{~cm}^{2} / \mathrm{s}$, which is significantly lower than values observed for conventional IEMs under the same conditions, despite the higher water and 
lower charge contents of these DNHs. Upon closer inspection, a thin exterior layer $(\sim 10-20 \mu \mathrm{m})$ was observed (Figure S10 in the SI). These "skin" layers encased both the top and bottom surfaces of the DNHs. The layers were hypothesized to arise during the formation of the second network and to be comprised primarily of the more hydrophobic second network. Analysis of the intrinsic transport properties of the bulk DNHs required removal of these skin layers, which was accomplished via vibratoming (Membrane Vibratoming section in the SI for further details). Characterizing various slices with attenuated total reflectance Fourier transform infrared (ATR FTIR) spectroscopy revealed a large reduction in the broad peak at $\sim 3,500 \mathrm{~cm}^{-1}-$ attributed to the $\mathrm{O}-\mathrm{H}$ stretch of water - when examining interior versus surface slices of a DNH sample (Figure S11 in the SI). The lower signal attributed to water is consistent with the hypothesis that the skin layers contain little-to-no hydrophilic poly(AMPS) material. Furthermore, SEM images of freezedried samples with the skin layer present were more uniform (non-porous) relative to interior sections, which can be used to rationalize the low salt permeability for these samples. Upon removal of the skin layer, the measured salt permeability of $0.1 \mathrm{~mol} / \mathrm{L} \mathrm{NaCl}$ for $95 \mathrm{~mol} \% \mathrm{EA}$ samples was $1.0 \times 10^{-6} \pm 10^{-7} \mathrm{~cm}^{2} / \mathrm{s}$ (Figure 6a), which is comparable to other membrane materials having similar water contents as discussed below ${ }^{24}$.

Salt permeability of the vibratomed DNH films was measured as a function of vol\% EA in the second network precursor solution. Generally, permeability decreased with increasing EA content, with the 100 vol\% EA samples showing a slight increase relative to the 95 vol\% EA samples (Figure 6a), consistent with the observed water content behavior (Figure 3). Inspired by Yasuda's free volume theory for hydrogels ${ }^{25}$ along with other reports on solute transport in polymer networks ${ }^{25,26,27}$, salt permeability was correlated with the inverse of water volume fraction and compared to uncharged and charged membrane materials (Figure 6b) ${ }^{24,28}$. The 
permeability of the DNH membranes prepared in this study compares favorably to uncharged cross-linked poly(ethylene glycol)-based membranes reported in the literature ${ }^{24}$ that are likely less mechanically sound (e.g., brittle) given their highly cross-linked single network architecture. Moreover, the DNHs prepared here show $\sim 8 \times$ lower salt permeability compared to measurements of the original DNHs (poly(AMPS)/poly(acrylamide)), which was prepared following a similar procedure to that reported by Gong and coworkers ${ }^{17}$.
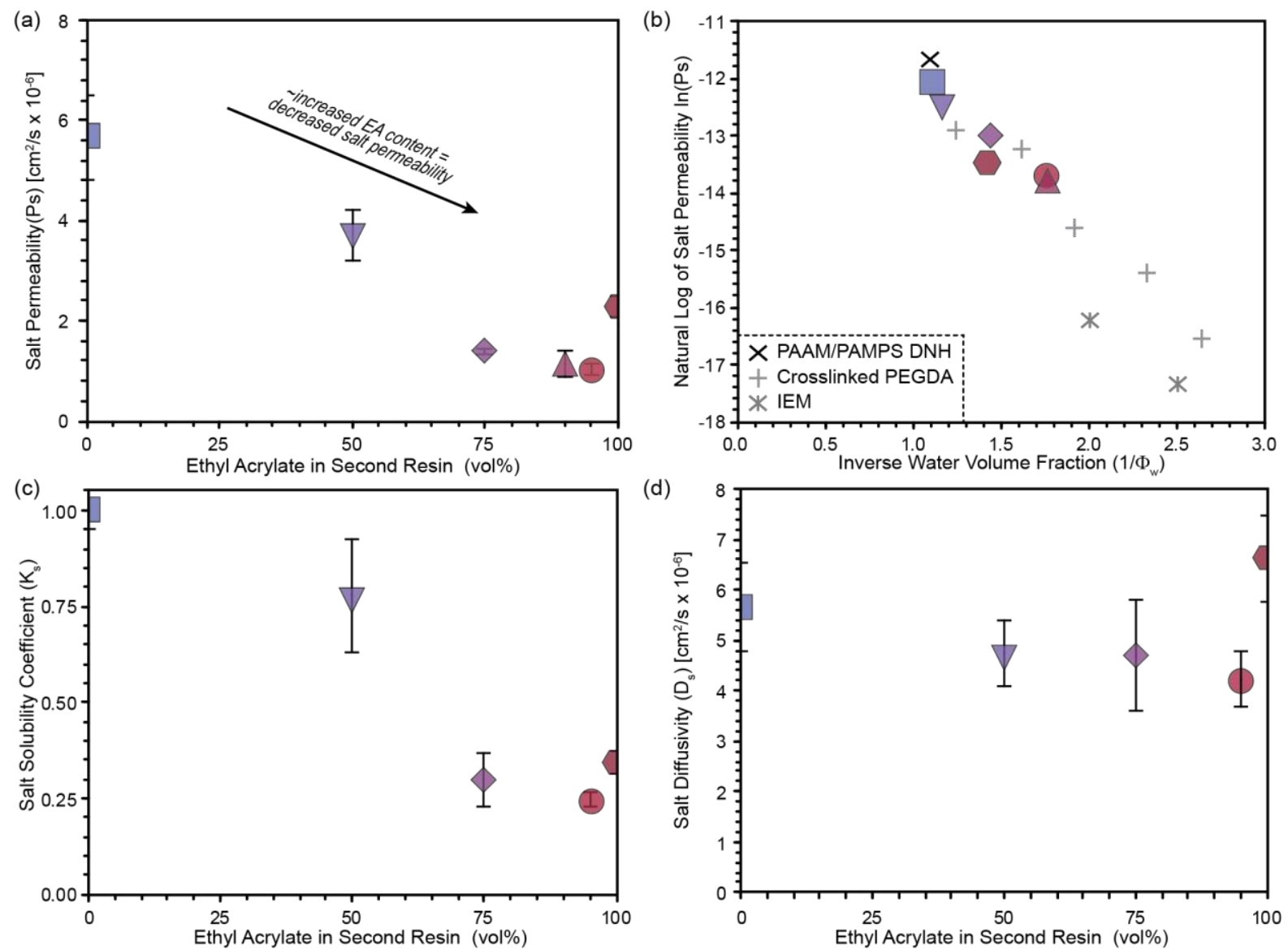

Figure 6. Salt transport properties. Salt permeability of the DNHs prepared in this study measured using $0.1 \mathrm{M} \mathrm{NaCl}$ as a function of (a) EA content and (b) inverse water volume fraction with other membrane materials shown for comparison. Salt solubility (c) and salt diffusivity (d) reported as a function of EA content. Values and error bars represent average and standard deviation from measurements on at least 3 separate samples, with the error bars for diffusivity calculated using standard propagation of error.

To better understand the reduction in salt permeability observed as the chemical composition of the second network was altered, salt solubility was measured, and salt diffusivity 
was extracted via the solution diffusion model ${ }^{29}$. Salt solubility is expressed as $K_{s}=C_{m} / C_{s}$, where $\mathrm{C}_{\mathrm{m}}$ and $\mathrm{C}_{\mathrm{s}}$ are the concentration of salt in the membrane (mol/L swollen membrane) and solution, respectively. Experimental details regarding solubility measurements are provided in the SI (Salt Solubility SI page S12). Figure $6 \mathbf{c}$ and $6 \mathbf{d}$ report salt solubility and diffusivity as a function of vol\% EA in the second network precursor solution, respectively. The reduction in salt solubility with increasing EA is consistent with the observed water uptake and charge content behavior. Membranes with lower water content tend to exhibit lower salt sorption, as the thermodynamic penalty for ions to partition into the polymer is increased. Moreover, membranes with higher charge content are able to electrostatically exclude mobile salt more efficiently. The decreasing trend observed for salt diffusivity between 0 and 95 vol\% EA is qualitatively consistent with free volume theory, based on reductions in water content. However, the small changes are within the measurements associated error. The similarity in diffusivity values suggests that the reduced salt permeability as EA content is increased primarily arises from decreased salt sorption. The origin of the relatively high salt diffusivity for the 100 vol\% EA is unknown, but is hypothesized to be related to differences in interactions between the pure EA second network and the hydrophilic AMPS network. In any case, this observation will serve as the basis for future studies. Given the observed trends and how commercial IEMs compare to these DNHs, we anticipate that further decreases in water content with concurrent increases in charge content will grant access to freestanding IEMs without the need for a fabric mesh support.

\section{Conclusion}

A systematic series of new DNHs were synthesized using a charged first network (AMPS) and a co-polymer second network consisting of a hydrophobic monomer (EA), to reduce water content, and a hydrophilic monomer (DMA) to stabilize the second network in water. Increasing EA 
content in the second network resulted in a decrease in water uptake to $\sim 1.1 \mathrm{~g}$ sorbed water/g dry polymer and an increase in charge density. Tensile testing revealed an increase in mechanical performance for DNHs prepared with larger amounts of EA (, in both stress $(\sim 3.5 \times)$ and strain $(\sim 4.5 \times)$ at break for 90:5 EA:DMA relative to pure DMA. Moreover, the strain energy density of these materials surpasses those of conventional IEMs, suggesting that the present DNHs may have the mechanical integrity required for operation as freestanding membranes. These results demonstrate that water content in DNHs can be reduced without compromising mechanical performance by using more hydrophobic second networks and solvents, such as NMF, that promote the partitioning of these second networks into the first network. Despite this advance, the fixed charge content of the DNHs remained relatively low compared to commercial IEMs, presumably due to an inherently low mass fraction of the first anionic network relative to the second neutral network. Salt transport properties relevant to water purification (i.e., permeability, solubility, and diffusivity) were measured using $0.1 \mathrm{M} \mathrm{NaCl}$ and placed into context with various membrane materials, including those with industrial relevance. These salt transport measurements revealed a general decrease in salt permeability as EA content was increased, which is qualitatively consistent with the observed water uptake data and free volume theory. Salt permeability of the DNHs prepared in this study was comparable to poly(ethylene glycol) membranes having similar water content $(\sim 60 \mathrm{wt} \%)$ and substantially reduced relative to traditional DNHs with high (>90 $\mathrm{wt} \%$ ) water content. These reductions in permeability are primarily attributed to reductions in salt solubility, though salt diffusivity decreased slightly as well. Ongoing synthetic efforts are focused on increasing charge content without substantially increasing water content via modified synthetic methods, including one pot preparation of DNHs and post-polymerization functionalization routes. 


\section{Acknowledgements}

This material is based upon work supported by the National Science Foundation under Grant No. DMR-2045336. For partial financial support we thank the Robert A. Welch Foundation under Grant No. F-2007 (Z. A. P.) and Grant No.F-1924-20170325 (R.S. and B.D.F.). This work was supported as part of the Center for Materials for Water and Energy Systems (M-WET), an Energy Frontier Research Center funded by the U.S. Department of Energy, Office of Science, Basic Energy Sciences under Award \#DE-SC0019272 (R. S. and B. D. F.). This material is based upon work supported by the National Science Foundation Graduate Research Fellowship under Grant No. DGE-1610403 (M. J. A.). The authors acknowledge the use of shared research facilities supported in part by the Texas Materials Institute. The authors would also like to thank James Burrow for his advice and help in performing SEM characterization. M. J. A. and R. S. contributed equally to this work.

\section{References}

1. Dragan, E. S. Chem. Eng. J., 2014, 243, 572-590.

2. Qadri, M. F., Malviya, R., Sharma, P. K. Open Pharm. Sci. J., 2015, 2, 21-30.

3. Pan, J., Zhu, L., Han, J., Hickner, M. A. Chem. Mater., 2015, 27, 6689-6698.

4. Ma, J., Zhou, G., Chu, L., Liu, Y., Liu, C., Luo, S., Wei, Y. ACS Sustain. Chem. Eng., 2017, 5, 843-851.

5. Gong, J. P., Katsuyama, Y., Kurokawa, T., Osada, Y. Adv. Mater., 2003, 15, 1155-1158.

6. Gong, J. P. Soft Matter, 2010, 6, 2583.

7. $\quad$ Chen, Q., Chen, H., Zhu, L., Zheng, J. J. Mater. Chem. B, 2015, 3, 3654-3676.

8. Nonoyama, T., Gong, J. P. 2021, 1-18.

9. Chen, Q., Chen, H., Zhu, L., Zheng, J. Macromol. Chem. Phys., 2016, 217, 1022-1036.

10. Haque, M. A., Kurokawa, T., Gong, J. P. Polymer, 2012, 53, 1805-1822.

11. Geise, G. M., Paul, D. R., Freeman, B. D. Prog. Polym. Sci., 2014, 39, 1-42.

12. Geise, G. M., Lee, H.-S., Miller, D. J., Freeman, B. D., McGrath, J. E., Paul, D. R. J. Polym. Sci. Part B Polym. Phys., 2010, 48, 1685-1718.

13. Mann, B. A., Holm, C., Kremer, K. J. Chem. Phys., 2005, 122, 154903.

14. Kamcev, J., Freeman, B. D. Annu. Rev. Chem. Biomol. Eng., 2016, 7, 111-133.

15. Ran, J., Wu, L., He, Y., Yang, Z., Wang, Y., Jiang, C., Ge, L., Bakangura, E., Xu, T. $J$. Memb. Sci., 2017, 522, 267-291.

16. Ducrot, E., Chen, Y., Bulters, M., Sijbesma, R. P., Creton, C. Science, 2014, 344, 186-189.

17. Matsuda, T., Nakajima, T., Gong, J. P. Chem. Mater., 2019, 31, 3766-3776.

18. Kundu, S., Simon, L. C., Fowler, M., Grot, S. 2005.

19. Genova-Dimitrova, P., Baradie, B., Foscallo, D., Poinsignon, C., Sanchez, J. Y. J. Memb. Sci., 2001, 185, 59-71.

20. Zhao, J., Guo, L., Wang, J. J. Memb. Sci., 2018, 563, 957-968.

21. Kamcev, J., Paul, D. R., Freeman, B. D. Macromolecules, 2015, 48, 8011-8024.

22. Strathmann, H. Ion-Exchange Membrane Separation Processes, 2004.

23. Landsman, M. R., Sujanani, R., Brodfuehrer, S. H., Cooper, C. M., Darr, A. G., Davis, R. J., Kim, K., Kum, S., Nalley, L. K., Nomaan, S. M., Oden, C. P., Paspureddi, A., Reimund, K. K., Rowles, L. S., Yeo, S., Lawler, D. F., Freeman, B. D., Katz, L. E. Annu. Rev. Chem. Biomol. Eng., 2020, 11, 559-585.

24. Ju, H., Sagle, A. C., Freeman, B. D., Mardel, J. I., Hill, A. J. J. Memb. Sci., 2010, 358, 131141. 
25. Yasuda, H., Lamaze, C., Ikenberry, L. D. Die Makromol. Chemie, 1968, 118, 19-35.

26. Dischinger, S. M., Gupta, S., Carter, B. M., Miller, D. J. Ind. Eng. Chem. Res., 2020, 59, 5257-5266.

27. Xie, W., Cook, J., Park, H. B., Freeman, B. D., Lee, C. H., McGrath, J. E. Polymer, 2011, 52, 2032-2043.

28. Kamcev, J., Paul, D. R., Manning, G. S., Freeman, B. D. ACS Appl. Mater. Interfaces, 2017, 9, 4044-4056.

29. Paul, D. R. J. Memb. Sci., 2004, 241, 371-386. 